\title{
Cingulate abnormalities associated with PANSS negative scores in first episode schizophrenia
}

\author{
Liz Ashton $^{\mathrm{a}}$, Anna Barnes ${ }^{\mathrm{b}, *}$, \\ Martin Livingston ${ }^{\mathrm{a}}$, David Wyper ${ }^{\mathrm{b}}$ and \\ The Scottish Schizophrenia Research Group \\ ${ }^{a}$ Department of Psychiatry, Southern General \\ Hospital, Glasgow, UK \\ ${ }^{\mathrm{b}}$ Institute of Neurological Sciences, Southern General \\ Hospital, Glasgow, UK
}

There is evidence for the involvement of the cingulate gyrus in schizophrenia. We present details of a Statistical Parametric Mapping (SPM) analysis of SPECT data from the largest study $(N=39)$ of drug naive schizophrenic patients. The main findings are that there is decreased perfusion in the anterior cingulate during verbal fluency when patients are compared to controls (matched individually by age, gender and father's social class as determined by occupation) and also that PANSS negative scores correlate negatively with regional cerebral blood flow in the cingulate gyrus (Pearson's Correlation coefficient of $r=-0.49$ and significance $p<0.005$ ). This suggests that measurement of change of perfusion in this region could be a useful biological marker in assessing the effect of neuroleptics on negative symptoms.

\section{Introduction}

Although neurochemical PET (Positron Emission Tomography) or SPECT (Single Photon Emission Tomography) tracers sensitive to specific aspects of synaptic function are now being used increasingly in schizophrenia to study both baseline functional abnormalities and the effects of neuroleptic medication, studies of regional functional activity using the more ubiquitous cerebral blood flow tracers can also be of value in detecting abnormal functional pathways. There are

*Corresponding author: Dr. A. Barnes, Department of Clinical Physics, Southern General Hospital, 1345 Govan Road, Glasgow G51 4TF, UK. many compounding factors in such studies. The imaging technique [PET or SPECT], the characteristics of the imaging camera, the tracer used, the stage of the illness, current and previous medication, and the mental activity and state of arousal during the trapping of the SPECT perfusion tracer can all influence the results. The last of these factors is perhaps the most difficult to control. Many investigators attempt to standardise the likely intellectual activity of the individual so that comparisons can be made between individuals and for the same individual investigated at different stages in the disease process. A degree of standardisation may be achieved through ensuring that the subject is in a resting state in a controlled environment during the uptake phase of the tracer. However this approach cannot control for the internal thought processes of the subject. In schizophrenia this could include a high level of anxiety, sometimes with hallucinations and frequently with delusional ideas. These factors may all influence the regions of the brain which are activated. In order to standardise to some extent, the use of activated challenge conditions has become more common place, with the most frequently used techniques being those of the Wisconsin Card Sort Test (WCS) or simple verbal fluency tests. These have the advantage of standardising the likely intellectual activity of subjects although it is far from clear whether the different test paradigms permit direct comparison between different study populations.

The most reported finding in functional neuroimaging studies of schizophrenia is that of hypofrontality although the definition of hypofrontality is often inconsistent, in particular in respect of frontal regions involved and whether it refers to reduced resting perfusion or reduced activation during a frontal task. Seminal work on this took place in the late nineteen eighties and early nineties and used ${ }^{133} \mathrm{Xe}$ SPECT. Weinberger et al. [41] investigated a group of 20 chronic schizophrenic patients who were all drug free for a pe- 
riod of at least 4 weeks prior to the study and found that there is evidence to support both definitions of hypofrontality. They found that schizophrenic subjects had lower relative frontal perfusion during rest compared to controls and also had reduced activation. They also found that the effect appeared to be largely independent of medication status and state factors such as attention, mental effort, or severity of psychotic symptoms [5]. On activation tests using the WCS, Weinberger et al found that reduced left frontal $\mathrm{rCBF}$ (regional Cerebral Blood Flow) correlated with poor task performance [40] and similar findings have been demonstrated using word-fluency activation with HMPAO SPECT in a group of twenty-five male, righthanded, medicated schizophrenic patients [23]. This study also found that negative symptom scores correlated inversely with mesial frontal $\mathrm{rCBF}$, particularly on the left. Andreasen et al. [2] used a different form of activation, the Tower of London task, which they found to be a relatively specific stimulant of the left mesial frontal cortex (probably including parts of the cingulate gyrus) in healthy normal volunteers. They included 13 neuroleptic-naive schizophrenic patients, 23 non-naive schizophrenic patients who had been relatively chronically ill but were medication free for at least 3 weeks, and 15 healthy normal volunteers in their study. They found decreased activation only in the patients with high scores for negative symptoms and suggested that hypofrontality is related to negative symptoms and is not a long-term effect of neuroleptic treatment or of duration of illness.

The study reported here used 39 exclusively neuroleptic naive schizophrenic patients with a tightly selected control group matched for age, gender and father's social class [36]. Imaging was performed on a high resolution SPECT scanner with the perfusion tracer ${ }^{99 \mathrm{~m}}$ Tc-HMPAO. The study was undertaken in 1993-1994 and the method of image analysis used in that report was based on visual determination of regions of interest [ROIs] which were selected using each subject's individual anatomical map determined by magnetic resonance imaging. This analysis showed that there was a loss of frontal asymmetry in schizophrenic men compared to controls with patients demonstrating hypofrontality. Since completing the study we have adapted and validated the Statistical Parametric Mapping (SPM) image analysis method developed for PET at the Wellcome Department of Cognitive Neurology [10]. This more powerful analysis method removes the constraints imposed by determination of ROIs. In particular we aimed to use the covariate analysis prop- erty of SPM to assess the relationship between function related regional perfusion and clinical variables. As the alleviation of negative symptoms is a focus in the development of many novel antipsychotic agents we were particularly interested in the relationship between regional perfusion and the Positive and Negative Syndrome Scores (PANSS) [3].

\section{Methods}

\subsection{Patients}

40 patients (32 Male, 8 Female) were selected from routine admissions to collaborating psychiatric units throughout Scotland. They were consecutive presentations to the psychiatric services who fulfilled the diagnostic criteria for either schizophrenia or schizophreniform psychosis according to DSM-III-R [1], were in their first episode of illness and were all drug naive. Patients were excluded if they had any other psychiatric disorder, a significant drug and alcohol history, head injury, or any significant physical disease. Informed consent was obtained from both patients and controls. Initial examination included assessment of psychopathology by means of the Positive and Negative Syndrome Scale (PANSS) [20], a urine drug screen, the Scottish Drinking Habits Questionnaire and, T1 and T2 weighted MRI scans to exclude organic brain pathology. Of these 40 patients 39 went on to have a ${ }^{99 \mathrm{~m}}$ Tc HMPAO scan and one patient was excluded on evidence of a pathological abnormality in the MRI scan.

\subsection{Controls}

Of the 40 normal control subjects recruited 39 went on to have a ${ }^{99 \mathrm{~m}} \mathrm{Tc}$ HMPAO SPECT scan. The controls matched the schizophrenic patients individually by age, gender and father's social class as determined by occupation. The controls had a full neurological examination and the same exclusion criteria were applied.

The experimental protocol was approved by the Ethics Committees of several Scottish hospitals including the Southern General Hospital where the SPECT and MRI scanning were undertaken. 


\subsection{Image acquisition}

All images were acquired using the SME 810 (Strichman Medical Equipment) dedicated SPECT system. For a full description of this scanner see Stoddart and Stoddart [39]. The patient scans were acquired using $500 \mathrm{MBq}{ }^{99 \mathrm{~m}}$ Tc HMPAO, slice thickness was $12 \mathrm{~mm}$, scanning time was 120 secs per slice, and slice spacing $6 \mathrm{~mm}$. The control scans were acquired using $250 \mathrm{MBq}$ ${ }^{99 \mathrm{~m}}$ Tc HMPAO, slice thickness was $12 \mathrm{~mm}$, scanning time was 240 secs per slice, and a slice spacing of $8 \mathrm{~mm}$. The lower dose was used for controls in order to comply with radiation regulations which require use of the lowest practicable exposure. The reduction in administered activity was partially compensated for by increasing the data acquisition time for the control subjects who were better able to accommodate this. These acquisition parameters provided an in-plane resolution of $8 \mathrm{~mm}$ and longitudinal resolution of $12 \mathrm{~mm}$. For each subject ${ }^{99 \mathrm{~m}} \mathrm{Tc}$ HMPAO was injected intravenously 1 minute into a verbal fluency task. Image acquisition began approximately 15 mins after injection with a total acquisition time of 40-60 mins depending on the slice thickness acquisition parameter and the size of the subject's head. Scan orientation was approximately parallel to the orbito-meatal (OM) line and head movement during the scanning procedure was minimised by subject co-operation and assisted by placing foam supports held in place by Velcro on either side of the subject's head. After reconstruction all scans were transferred to a SUN SPARCULTRA 10 workstation (Sun Microsystems, Camberley, UK) for further processing and analysis.

\subsection{Verbal fluency task}

A verbal fluency task [4] was used to produce a degree of standardisation of the cognitive state of subjects during the period of fixation of the tracer and to accentuate task related group differences. The task was performed in a quiet room, with the subject's eyes closed. Subjects were given a letter (letters used were F, A, R, $\mathrm{L}, \mathrm{S}, \mathrm{C}$ ) and asked to say out loud as many words as possible beginning with that letter for 1 minute. The task began 1 minute before and continued until 5 minutes following intravenous administration of tracer.

\subsection{Image processing}

All scans were processed using the statistical parametric mapping (SPM) module in MEDx3.0 (Sensor Systems, Inc., Virginia, USA) which is a $\mathrm{C}+$ analogy of the MATLAB (Mathworks Inc., Cambridge Control, UK) based SPM96 (Wellcome Department of Cognitive Neurology, London, UK. http://www.fil.ion.bpmf.ac.uk/spm) image analysis software.

All the scans were spatially normalised to the PET CBF template provided with MEDx3.0 using the linear spatial normalisation option. All spatially normalised scans were smoothed using a $12 \mathrm{~mm} \mathrm{FWHM} \mathrm{(full} \mathrm{width}$ at half the maximum) isotropic Gaussian spatial filter prior to the voxel-wise statistical analysis. Global voxel intensity normalisation was achieved using proportional scaling so that any differences in global cerebral blood flow (gCBF) were removed by scaling all voxels within a scan to the mean $\mathrm{gCBF}$ of that scan.

\subsection{Image analysis}

All scans were statistically analysed using the appropriate SPM module in MEDx3.0. This type of voxelwise analysis is documented elsewhere for neuroactivation studies [10]. In brief it consists of: a) An image co-registration facility to correct for differences in yaw, roll and pitch; b) spatial normalisation using a 12 parameter linear transformation into Talairach coordinate space using scaling and shearing to account for differences in individual brain shape (i.e. stereotactic anatomic standardisation) and c) voxelwise statistical parametric analysis based on the theory of Gaussian fields and using global mean blood flow to normalise voxel activity. The resulting statistical parametric maps $(\operatorname{SPM}(\mathrm{t}))$ represent a map of change significance based on

- peak effect size $(Z)$ within a contiguous volume with voxel values greater than $Z=2.33$

- the size $\mathrm{V}$ of this volume

- the smoothness of the data-set

- the overall size of the search volume.

The group differences, controls compared to patients, were analysed using the statistics option "compare groups: 1 scan per subject". A further investigation of the patients' scans was performed using a covariates only analysis where the positive and negative symptom (PANSS) scores for each patient were used as covariates of interest and correlated to $\mathrm{rCBF}$. 


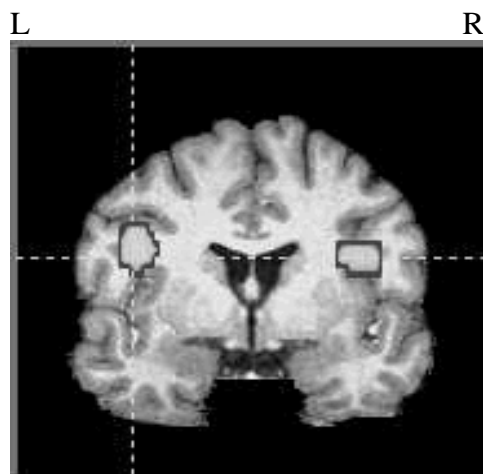

a)

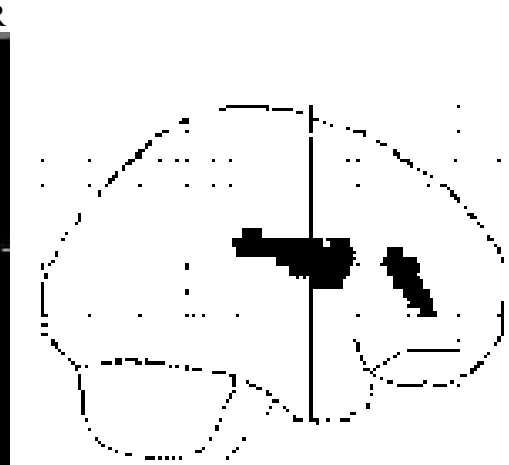

b)

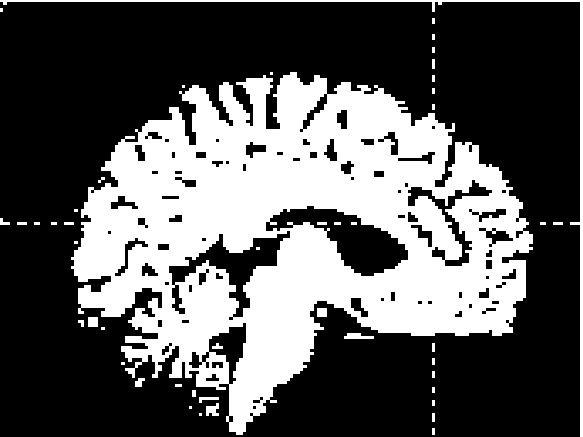

c)

Fig. 1. A statistical parametric map of significant CBF decreases (anterior cingulate, $K=138$ voxels, $Z$ max $=3.74(x, y, z)=2,32,24$, BA 32 and bilateral pre-motor cortex, $K=230$ voxels, $Z \max =4.22,(x, y, z)=-46,-6,28$, BA $6 / 9 ; K=223$ voxels, $Z \max =4.05(x, y, z)=40,6,24$, BA 6/9) in the first episode schizophrenic patients compared with controls is shown in Fig. 1(b). This is superimposed onto an anatomically standardised T1 MR image to facilitate visualisation (Figs (a) and (c)).

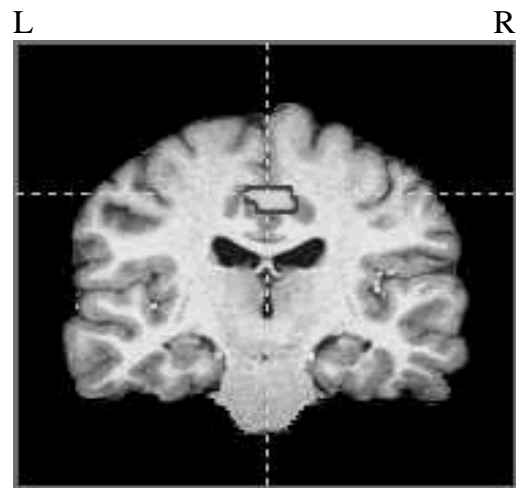

a)

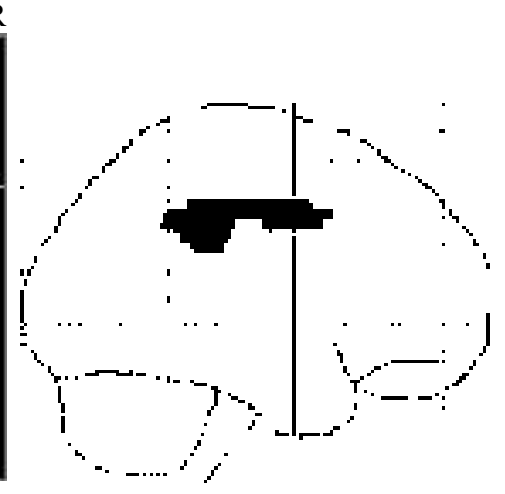

b)

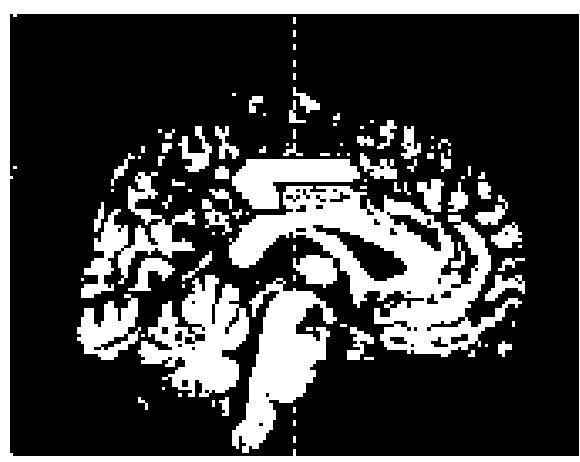

c)

Fig. 2. A statistical parametric map of the significant area of decreased CBF (cingulate, $K=575$ voxels, $Z$ max $=3.19, r=-0.49$, $(x, y, z)=-4,-40,40$, BA 32/24/31) associated with increased negative symptom score (PANSS negative) in first episode schizophrenic patients is shown in Fig. 1(b). This is superimposed onto an anatomically standardised T1 MR image to facilitate visualisation (Figs (a) and (c)).

\section{Results}

The mean scores on fluency testing were 77.72 (range 38-168) for controls and 59 (range 1-111) for patients (with the controls performing significantly better $P=0.013)$. Although detailed assessment of mental state was not taken at the time of scanning, no patient was observed to be experiencing hallucinations at this time. The blood flow differences between controls and the first episode schizophrenic patients are displayed in the SPM(t) map (Fig. 1), with thresholds set at $P u<0.001$ (uncorrected height threshold) and $P k<0.3$ (corrected extent threshold), where 'height' is the voxel intensity within the $\operatorname{SPM}(\mathrm{t})$ and 'extent' is the number of voxels above this height threshold cor- rected for multiple statistical testing [33]. The map shows a decrease in blood flow in the patient scans with respect to the control scans in the area of the anterior cingulate (Brodmann area 32) and bilaterally in the pre-motor cortex (Brodmann areas 6/9).

A within group correlation analysis in the patient group only tested for a significant relationship between $\mathrm{CBF}$ and PANSS scores. The results of the correlation of CBF with PANSS negative score are displayed in the SPM(t) map (Fig. 2), with thresholds set at $P u<0.005$ (uncorrected height threshold) and $P k<0.1$ (corrected extent threshold) and show a negative correlation of rCBF with negative symptom score with a Pearson's Correlation coefficient of $r=-0.49$ and significance $p<0.005$ for the most significant voxel. The map 
shows a region of decrease in blood flow corresponding to an increase in PANSS negative score in patients in the area of the cingulate (Brodmann areas 32, 24 (anterior cingulate) and Brodmann area 31 (posterior cingulate)).

An analysis of CBF correlated with PANSS positive score failed to show any significant relationship and there was no significant relationship between fluency scores and PANSS negative scores (Pearson's Correlation coefficient $r=0.1097$ ).

\section{Discussion}

\subsection{Image acquisition}

Different image acquisition protocols were used for patients and controls in this study. Patients had a full $500 \mathrm{MBq}$ injection of ${ }^{99 \mathrm{~m}} \mathrm{Tc}-\mathrm{HMPAO}$ while controls received half this amount. This was necessitated by the radiation guidelines on investigation of controls. It was partially compensated for by increasing the data acquisition time for the control subjects who were better able to accommodate this and by increasing the slice spacing of the section scanner from $6 \mathrm{~mm}$ to $8 \mathrm{~mm}$. It should be noted, however, that the image longitudinal spatial resolution is governed primarily by the collimator geometry and that slice spacing is a second order effect. The final image quality did not differ noticeably between the groups and the spatial resolution was around $8 \mathrm{~mm}$ transverse and $12 \mathrm{~mm}$ longitudinal for both groups. It is unlikely that the subtle difference in acquisition protocols could account for differences in any particular focal region. It should also be noted that the finding that perfusion in the cingulate gyrus is negatively correlated to PANSS negative scores does not use the control data and so is unaffected by the group difference in image acquisition protocol.

\subsection{Data analysis}

Original analysis of our data [36] using a regions of interest (ROI) method failed to show significant differences in blood flow in the cingulate when comparing patients with controls. This inconsistency between the two methods of analysis may be due to certain limitations associated with the use of ROIs. Large ROIs may lump functionally distinct areas together and so mask changes or abnormalities affecting only part of the ROI or if a significant change is detected the ROI may cross functional boundaries hiding the true loca- tion of the change. The size and shape of the region is also important since an ROI that conforms precisely to the shape of the structure will detect a change with greater sensitivity than a geometrically regular shape. In both cases the placing of an ROI is entirely dependent on the investigator's ability to identify brain structures and a degree of expertise in human neuroanatomy is needed for this. The use of voxel-wise techniques such as SPM, where the regions are effectively reduced to single voxels, remove these problems of observer bias. However, the limitations of using voxel-wise methods for the analysis of functional brain images is that they are very sensitive to any pre-processing of the images and underlying statistical assumptions made about this data. The user therefore must ensure the consistency of all pre-processing steps of the images as well as having a thorough understanding of the statistical methods and inferences used on the data. A more in depth discussion comparing ROI analysis with SPM can be found in Montaldi et al. [28] and discussion of SPM analysis and statistical inference is given in the paper by Friston et al. [12].

\subsection{Key findings}

This re-analysis of the data using SPM has highlighted two important findings. The first is that there is significantly reduced perfusion in the anterior cingulate and bilaterally in the pre-motor cortex comparing schizophrenics with controls during the performance of a verbal fluency task. As will be discussed below this is not a novel finding and is in agreement with the majority of evidence from previous imaging studies. The second finding is the association between negative symptoms and perfusion in the cingulate. These are discussed below.

\subsection{The role of the anterior cingulate in putative frontal lobe tasks}

The role of the anterior cingulate in verbal fluency and similar cognitive tasks has been thoroughly investigated. Studies using fMRI [31,32] and PET [7,8] have shown that the anterior cingulate (BA32) and/or the pre-motor areas (BA6) are activated in normal control subjects when performing a verbal fluency test. The fMRI study by Paulesu et al. [31] showed that there was activation of the premotor areas of the cortex in normal subjects while performing a verbal fluency task similar to that employed in this study, although they did not report activation in the area of the anterior cin- 
gulate. The other three studies showed activation of both the anterior cingulate and premotor areas in normal subjects with verbal fluency tests, some of which involved a memory component in addition to free recall. Fletcher et al. [8] extend their results using connectivity theory [9] to suggest that the anterior cingulate with the premotor areas form part of a network of interconnected regions involved in the fluency task, that may be disrupted in schizophrenia.

\subsection{The cingulate gyrus and schizophrenia}

The cingulate gyrus is part of the limbic system and has extensive reciprocal connections with cortical and sub-cortical structures. It is involved in motivation, memory and attention [29]. It has therefore come under scrutiny as a region of special interest to those investigating the neurological substrate of schizophrenia. There may be cyto-architectural changes in the upper layers of the anterior cingulate gyrus in patients with schizophrenia [19]. An MRI study of patients with schizophrenia and age, sex and socio-economically (by status of parents) matched normal controls [30] concluded that the schizophrenic subjects had significantly smaller cingulate volumes.

Our finding of a decrease in anterior cingulate perfusion in untreated schizophrenics compared with controls is consistent with the reduced metabolic rate for glucose found by Haznedar et al. [18] and Siegal et al. [38]. The study by Haznedar used fifty drug free schizophrenic patients, only 23 of whom were drug naive and the study by Siegel recruited seventy male schizophrenic patients, only 27 of whom were drug naive.

\subsection{Are we observing reduced function in the cingulate or reduced activation during the fluency task?}

In both the Haznedar and Siegel studies subjects performed cognitive tasks throughout the 35 minutes of the PET scan and so it is unclear whether the observed regional reductions in cerebral metabolic rate for glucose are baseline effects or are a result of reduced activation during the task. Our study cannot resolve this issue either. A baseline scan would have achieved this but, as it was not considered ethical to withhold treatment for the acute first episode patients, the split dose SPECT technique [37] would have to have been employed to enable both baseline and challenge scans to be obtained in a single session. This was judged to be undesirable due to the reduced image quality and increased data acquisition time associated with the split dose method. Mellers et al. [27], however, did use the split dose technique in a study similar to ours but focusing on the differences between schizophrenic and epileptic patients. They found an increased activation of regional blood flow in the anterior cingulate during the performance of a fluency task in the schizophrenic group. This contrasts with our finding of reduced cingulate perfusion in schizophrenics. The Mellers et al. study did, however, use medicated patients and did not have a normal control group but set out to compare schizophrenia with an epilepsy group and a group with schizophrenia-like psychosis of epilepsy (SLPE). In their study there was no significant difference in verbal fluency scores between schizophrenic and epilepsy patients but there was a significant increase in fluency scores in schizophrenics compared to the SLPE group. Most other papers have observed reduced cingulate activation during the performance of putative frontal tasks. In a PET study Crespo-Facorro et al. [7] found decreased blood flow activation in the right anterior cingulate in 14 medication free schizophrenics compared to 13 healthy controls during the performance of a novel memory task. Fletcher et al. [8] take this debate forward by proposing that the anterior cingulate may be a key region in the functional characterisation of schizophrenia in that it has a role in the modulation of prefronto-temporal (Brodmann areas 9/46) integration. In accordance with this 'connectivity' theory $[11,13,17,24]$ of schizophrenia is the theory that schizophrenia is a neurodevelopmental disorder with abnormal migration of neurones occurring in utero during the middle trimester of pregnancy $[17,34]$.

\subsection{Task performance}

Task performance can be constrained so that patient and control groups perform equally well (e.g., with a paced verbal fluency task). We elected not to constrain the task in order that fluency performance could be assessed and correlated with other parameters and also to avoid differences in effort. This does, however, present the problem that the observed cingulate activation could be due to reduced performance scores of the patient group. The literature reviewed above suggests, however, that this is not the main factor explaining the group differences. Importantly, in this study, task performance did not correlate with negative symptom rating and therefore task performance cannot account for the relationship which we found between cingulate perfusion and PANSS rating. 


\subsection{Cingulate function and clinical status}

The functioning of the cingulate has been linked with the clinical status of patients who have schizophrenia. In a SPECT study [35], which compared 24 never medicated schizophrenics who were drug free and neurologically intact, with 20 control subjects, areas of both hyperperfusion and hypoperfusion were found in the patients, all of whom were acutely ill. These blood flow patterns were associated positively and negatively with positive symptoms assessed by means of the PANSS [20] rating scale. One negative symptom correlated "negatively and strongly" with rCBF in the cingulate as well as left frontal, temporal and parietal regions. After antipsychotic drug treatment the correlation between the negative symptom and cingulate (as well as frontal, temporal, basal ganglia and thalamic) hypofunction persisted. We also found a negative correlation between $\mathrm{rCBF}$ in the cingulate and negative symptoms on the PANSS.

It is interesting that the negative relationship found in our study does not reach significance in the extreme anterior aspect of the cingulate as can be seen by contrasting Figs 1 and 2. This could mean that the anterior aspect is not involved in the processes associated with negative symptoms. An alternative interpretation of this finding is that the relationship is non-linear and the already reduced perfusion which we found in the anterior cingulate regardless of negative symptom score reduces the magnitude of change in that region.

In an extensive review of the literature, Buchsbaum and Hazlett [6] found evidence that the intensity of negative symptoms is associated with hypofrontality. Although the studies reviewed involved patients with various histories of medication and both the definition of 'intensity of symptoms' and the methods of analysing images differed, the weight of evidence supports a relationship between hypofrontality and negative symptoms. However, the regional specificity of hypofrontality perhaps needs to be defined in more detail. For example, the PET study by Siegal et al. [38] of 70 unmedicated schizophrenic patients found an inverse relationship between negative symptoms and medial frontal (including the cingulate) but not lateral frontal metabolic rate.

\subsection{The cingulate, negative symptoms and mood}

The interaction between mood and cognitive function has been studied in normal subjects with PET by Baker et al. [3]. They found that the activation of the cingulate gyrus associated with the performance of a fluency task was reduced in depressed mood conditions.

There is a considerable literature on the cingulate gyrus in depressive illness $[3,15,17,21,25,26]$ which appears to be contradictory. Some [3,6,15] show a decrease in $\mathrm{rCBF}$ to be associated with major depressive disorder (MDD) where others [21,25] show cingulate areas to be hyperactive in MDD. Mayberg [25] pulls these strands together with the finding that anterior cingulate hypometabolism predicts non-responders to medication in MDD whereas treatment responders show hypermetabolism of the anterior cingulate.

These findings are interesting because all current methods of assessing negative symptoms lack specificity. Assessors may be rating depression and movement disorder as well as negative symptoms of schizophrenia, apart from the technical problems that are inherent in determining the absence of some phenomenon in a person's mental state. Indeed the items of the PANSS for negative symptoms are also symptoms and signs sometimes found in MDD; blunted affect, emotional withdrawal, poor rapport, social withdrawal, difficulty in abstract thinking, lack of spontaneity and flow of conversation and stereotyped thinking. This could mean that our findings simply represent depressed mood at the time of assessment. In a study assessing depressive illness in schizophrenia [22], it was found that depressive symptoms were associated with delusional beliefs rather than negative symptoms, but whether there is a common neurobiological substrate linking the negative symptoms manifest in depression and schizophrenia is at present uncertain.

\subsection{The cingulate and dopamine}

Linkage between dopamine and cingulate functioning has been demonstrated [16]. All antipsychotic drugs have dopamine antagonistic properties in their profile of activity. A post-mortem study has shown that DOPAC, a dopamine metabolite, is reduced in the anterior cingulate of patients with schizophrenia compared with controls [42]. This finding appears to be unrelated to the time of harvesting the brain after death, length of time the specimen has spent in the freezer, the age of the subject or antipsychotic drug status. Furthermore, perturbation of the dopamine system in live subjects with schizophrenia impacts on cingulate functioning as shown by Frith et al. [14]. Apomorphine, a dopamine agonist was administered to patients with schizophrenia undergoing a PET scan while participating in a cognitive task. These were unmedicated schizophrenic 
patients and they displayed enhanced cognitive activation of the anterior cingulate cortex relative to normal subjects.

\subsection{Neuroleptic medication}

Negative symptoms in schizophrenia essentially amount to the absence of certain neural state functions such as motivation and volition. The assessment of these is highly subjective, whether carried out as part of a clinical interview or by means of a structured rating scale such as the PANSS. This can be a problem when evaluating the effect of antipsychotics on negative symptoms. As the alleviation of negative symptoms is a focus in the development of many novel antipsychotic agents, the relationship which we have shown here between cingulate function and the negative symptoms of schizophrenia suggests that change in cingulate perfusion could be a useful objective biological marker in the assessment of the effectiveness of these agents.

\subsection{Conclusion}

We present further evidence for the involvement of the cingulate gyrus in schizophrenia suggesting decreased activation of the anterior cingulate in drug naive patients undertaking a fluency task and we have found an association between negative PANSS scores and decreased rCBF in the cingulate gyrus. Change in cingulate function could be a useful objective biological marker in the assessment of neuroleptic medication on negative symptoms.

\section{Acknowledgements}

The Scottish Schizophrenia Research Group led by Dr R McCreadie and Dr Livingston collected the data used for this analysis and we would like to thank the patients, control subjects and all clinicians and scientists involved with the original study. Additional funding was supplied by the Robertson Trust.

\section{References}

[1] American Psychiatric Association, Diagnostic and Statistical manual of mental disorders, American Psychiatric Association, Washington, DC, 1987.

[2] Andreasen, N.C., Rezai, K. and Alliger, R., Hypofrontality in neuroleptic-naive patients and in patients with chronic schizophrenia. Assessment with xenon 133 single-photon emission computed tomography and the Tower of London, Archive of General Psychiatry 49 (1992), 943-958.
[3] Baker, S.C., Frith, C.D. and Dolan, R.J., The interaction between mood and cognitive function studied with PET, Psychological Medicine 27, 565-578.

[4] Benton, A.L., Differential effects in frontal lobe disease, $\mathrm{Neu}$ ropsycholgia 6 (1968), 53-60.

[5] Berman, K.F., Zec, R.F. and Weinberger, D.R., Physiologic dysfunction of dorsolateral prefrontal cortex in schizophrenia. II. Role of neuroleptic treatment, attention, and mental effort, Archive of General Psychiatry 43 (1986), 126-135.

[6] Buchsbaum, M.S. and Hazlett, E.A., Positron emission tomography studies of abnormal glucose metabolism in schizophrenia, Schizophrenia Bulletin 24 (1998), 343-364.

[7] Crespo-Facorro, B., Paradiso, S., Andreasen, N.C., O'Leary, D.S., Watkins, G.L., Boles Ponto, L.L. and Hichwa, R.D., Recalling word lists reveals "cognitive dysmetria" in schizophrenia: a positron emission tomography study, American Journal of Psychiatry 156 (1999), 386-392.

[8] Fletcher, P., McKenna, P.J., Friston, K.J., Frith, C.D. and Dolan, R.J., Abnormal cingulate modulation of frontotemporal connectivity in schizophrenia, Neuroimage $\mathbf{9}$ (1999), 337-342.

[9] Friston, K.J., Frith, C.D., Liddle, P. and Frackowiak, R.S.J., Functional connectivity: The principal component analysis of large (PET) data sets, Journal of Cerebral Blood Flow and Metabolism 13 (1993), 5-14.

[10] Friston, K.J., Holmes, A.P., Worsley, K.J., Poline, J.B., Frith, C.D. and Frackowiak, R.S.J., Statistical Parametric Maps in Functional Imaging: A general linear approach, Human Brain Mapping 12 (1995), 189-210.

[11] Friston, K.J. and Frith, C.D., Schizophrenia: a disconnection syndrome? Clinical Neuroscience 3 (1995), 89-97.

[12] Friston, K.J., Holmes, A., Poline, J.B., Price, C.J. and Frith, C.D., Detecting activations in PET and fMRI: levels of inference and power, Neuroimage 4 (1996), 223-235.

[13] Frith, C.D., Functional brain imaging and the neuropathology of schizophrenia [comment], Schizophrenia Bulletin 23 (1997), 525-527.

[14] Frith, C.D., Friston, K.J., Herold, S., Silbersweig, D., Fletcher, P., Cahill, C., Dolan, R.J., Frackowiak, R.S. and Liddle, P.F., Regional brain activity in chronic schizophrenic patients during the performance of a verbal fluency task, British Journal of Psychiatry 167 (1995), 343-349.

[15] Galynker, I.I., Cai, J., Ongseng, F., Finestone, H., Dutta, E. and Serseni, D., Hypofrontality and negative symptoms in major depressive disorder, Journal of Nuclear Medicine 39 (1998), 608-612.

[16] Glenthoj, B.Y. and Hemmingsen, R., Transmitter dysfunction during the process of schizophrenia, Acta Psychiatrica Scandinavaca Suppl 395 (1999), 105-112.

[17] Harrison, P.J., The neuropathology of schizophrenia. A critical review of the data and their interpretation, Brain 122 (1999), 593-624.

[18] Haznedar, M.M., Buchsbaum, M.S., Luu, C., Hazlett, E.A., Siegel, B.V., Jr., Lohr, J., Wu, J., Haier, R.J. and Bunney, W.E., Jr., Decreased anterior cingulate gyrus metabolic rate in schizophrenia, American Journal of Psychiatry 154 (1997), 682-684.

[19] Hecker, S., Neuropathology of schizophrenia: cortex, thalamus, basal ganglia and neurotransmitter specific systems, Schizophrenia Bulletin 23 (1997), 403-421.

[20] Kay, S.R., Fiszbein, A. and Opler, L.A., The positive and negative syndrome scale (PANSS) for schizophrenia, Schizophrenia Bulletin 13 (1987), 261-276. 
[21] Kishimoto, H., Yamada, K., Iseki, E., Kosaka, K. and Okoshi, T., Brain imaging of affective disorders and schizophrenia, Psychiatry and Clinical Neuroscience 52 (1998), S212-S214.

[22] Kohler, C., Swanson, C.L., Gur, R.C., Mozley, L.H. and Gur, R.E., Depression in schizophrenia: II. MRI and PET findings, Biological Psychiatry 43 (1998), 173-180.

[23] Lewis, S.W., Ford, R.A., Syed, G.M., Reveley, A.M. and Toone, B.K., A controlled study of 99mTc-HMPAO single photon emission imaging in chronic schizophrenia, Psycholological Medicine 22 (1992), 27-35.

[24] Liddle, P.F., Functional imaging-schizophrenia, British Medical Bulletin 52 (1996), 486-494.

[25] Mayberg, H.S., Brannan, S.K., Mahurin, R.K., Jerabek, P.A., Brickman, J.S., Tekell, J.L., Silva, J.A., McGinnis, S., Glass, T.G., Martin, C.C. and Fox, P.T., Cingulate function in depression: a potential predictor of treatment response, Neuroreport 8 (1997), 1057-1061.

[26] Mayberg, H.S., Liotti, M., Brannan, S.K., McGinnis, S., Mahurin, R.K., Jerabek, P.A., Silva, J.A., Tekell, J.L., Martin, C.C., Lancaster, J.L. and Fox, P.T., Reciprocal limbic-cortical function and negative mood: converging PET findings in depression and normal sadness, American Journal of Psychiatry 156 (1999), 675-682.

[27] Mellers, J.D., Adachi, N., Takei, N., Cluckie, A., Toone, B.K. and Lishman, W.A., SPET study of verbal fluency in schizophrenia and epilepsy, British Journal of Psychiatry 173 (1998), 69-74

[28] Montaldi, D., Mayes, A.R., Barnes, A., Pirie, H., Hadley, D.M., Patterson, J. and Wyper, D.J., Associative encoding of pictures activates the medial temporal lobes, Human Brain Mapping 6 (1998), 85-104.

[29] Mueser, K.T., Bellack, A.S., Douglas, M.S. and Wade, J.H., Prediction of social skill acquisition in schizophrenic and major affective disorder patients from memory and symptomatology, Psychiatry Research 37 (1991), 281-296.

[30] Noga, J.T., Aylward, E., Barta, P.E. and Pearlson, G.D., Cingulate gyrus in schizophrenic patients and normal volunteers, Psychiatry Research 61 (1995), 201-208.

[31] Paulesu, E., Goldacre, B., Scifo, P., Cappa, S.F., Gilardi, M.C., Castiglioni, I., Perani, D. and Fazio, F., Functional heterogeneity of left inferior frontal cortex as revealed by fMRI, Neuroreport 8 (1997), 2011-2017.

[32] Phelps, E.A., Hyder, F., Blamire, A.M. and Shulman, R.G.,
FMRI of the prefrontal cortex during overt verbal fluency, Neuroreport 8 (1997), 561-565.

33] Poline, J.B., Worsley, K.J., Evans, A.C. and Friston, K.J., Combining spatial extent and peak intensity to test for activations in functional imaging, Neuroimage 5 (1997), 83-96.

[34] Roberts, G.W., Schizophrenia: a neuropathological perspective, British Journal of Psychiatry 158 (1991), 8-17.

[35] Sabri, O., Erkwoh, R., Schreckenberger, M., Owega, A., Sass, H. and Buell, U., Correlation of positive symptoms exclusively to hyperperfusion or hypoperfusion of cerebral cortex in nevertreated schizophrenics, Lancet 349 (1997), 1735-1739.

[36] Scottish Schizophrenia Research Group, Regional cerebral blood flow in first-episode schizophrenia patients before and after antipsychotic drug treatment. Scottish Schizophrenia Research Group, Acta Psychiatrica Scandinavica 97 (1998), 440-449.

[37] Shedlack, K.J., Hunter, R., Wyper, D., McLuskie, R., Fink, G. and Goodwin, G.M., The pattern of cerebral activity underlying verbal fluency shown by split-dose single photon emission tomography (SPET or SPECT) in normal volunteers, Psychological Medicine 21 (1991), 687-696.

[38] Siegel, B.V., Jr., Buchsbaum, M.S., Bunney, W.E., Jr., Gottschalk, L.A., Haier, R.J., Lohr, J.B., Lottenberg, S., Najafi, A., Nuechterlein, K.H. and Potkin, S.G., Cortical-striatalthalamic circuits and brain glucose metabolic activity in 70 unmedicated male schizophrenic patients, American Journal of Psychiatry 150 (1993), 1325-1336.

[39] Stoddart, H.A. and Stoddart, H.F., New multi-dimensional reconstructions for the 12-detector, scanned focal point, singlephoton tomograph, Physics in Medicine and Biology 37 (1992), 579-586.

[40] Weinberger, D.R., Berman, K.F. and Illowsky, B.P., Physiological Dysfunction of Dorsolateral Prefrontal Cortex in Schizophrenia, Archive of General Psychiatry 45 (1988), 609615.

[41] Weinberger, D.R., Berman, K.F. and Zec, R.F., Physiologic dysfunction of dorsolateral prefrontal cortex in schizophrenia. I. Regional cerebral blood flow evidence, Archive of General Psychiatry 43 (1986), 112-124.

[42] Wyatt, R.J., Karoum, F. and Casanova, M.F., Decreased DOPAC in the anterior cingulate cortex of individuals with schizophrenia [see comments], Biological Psychiatry 38 (1995), 4-12. 


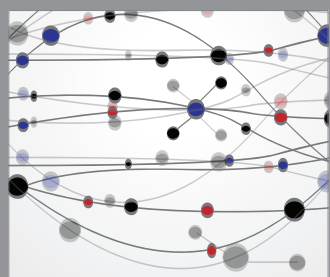

The Scientific World Journal
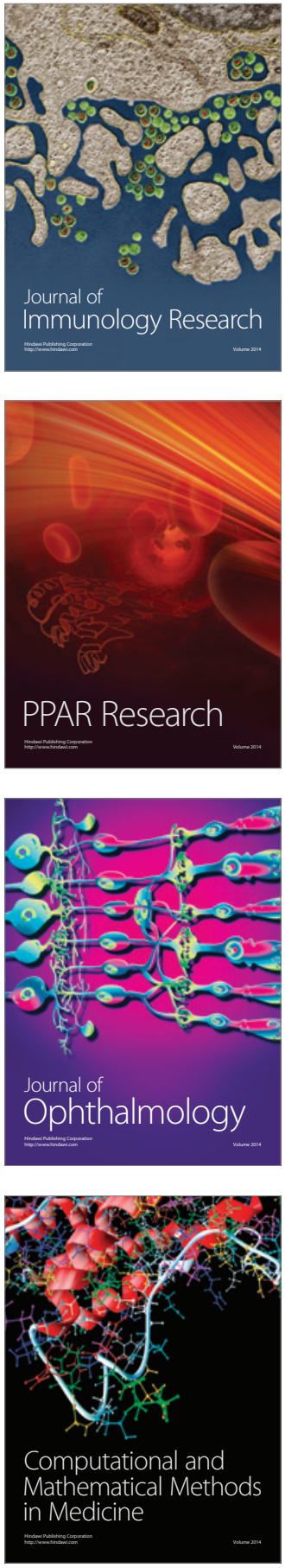

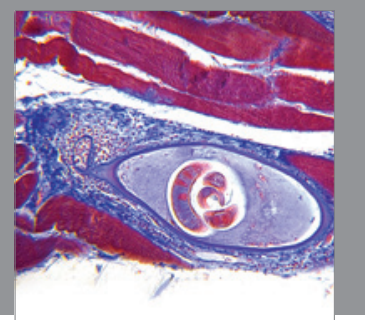

Gastroenterology

Research and Practice
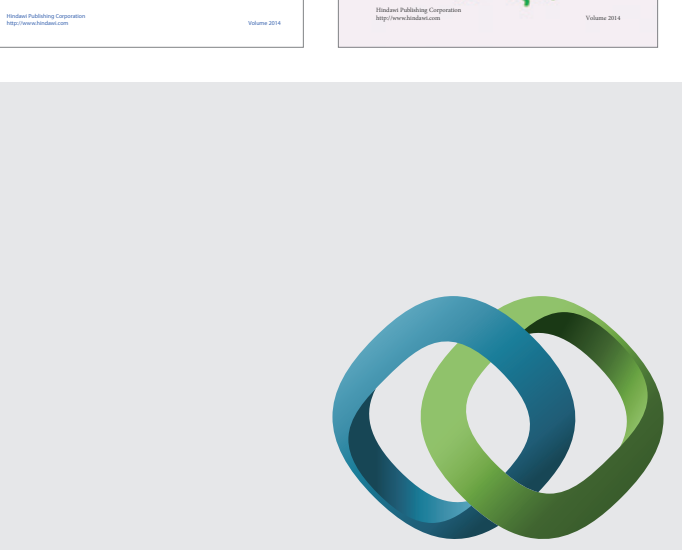

\section{Hindawi}

Submit your manuscripts at

http://www.hindawi.com
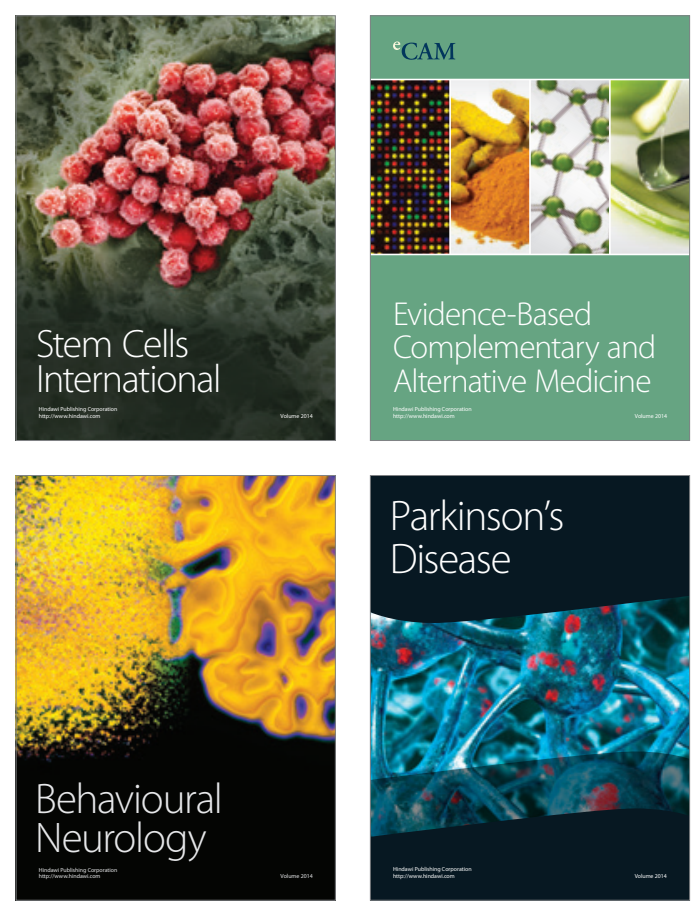

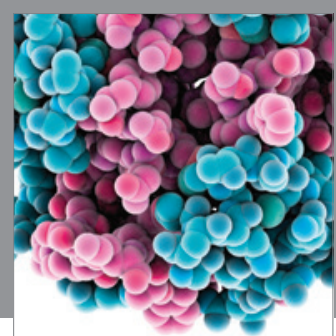

Journal of
Diabetes Research

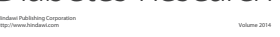

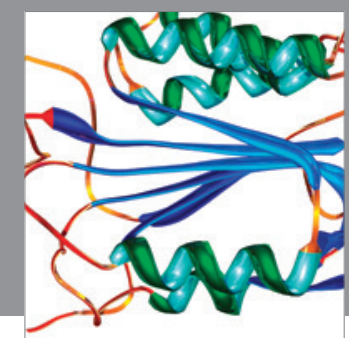

Disease Markers
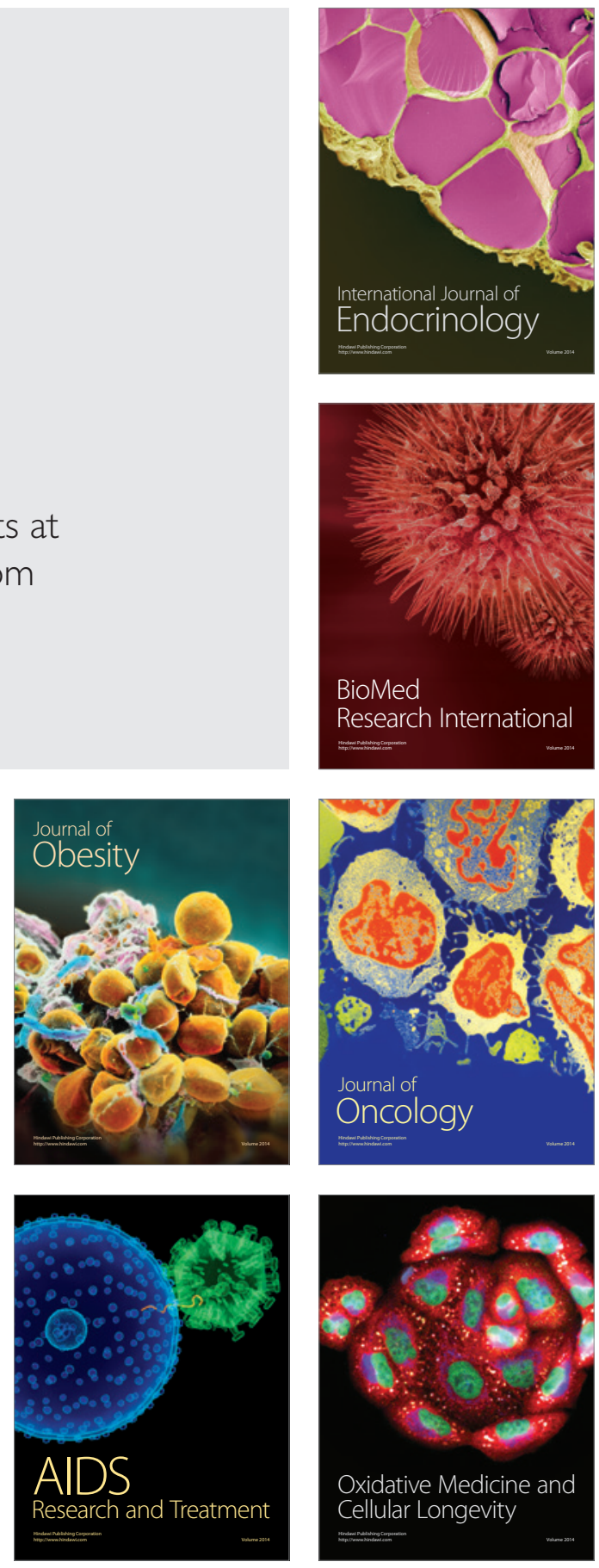